\title{
Central blood pressure: a powerful predictor of the development of hypertension
}

\author{
Hirofumi Tomiyama ${ }^{1}$, Michael F O'rourke ${ }^{2}$, Hideki Hashimoto ${ }^{3}$, Chisa Matsumoto ${ }^{1}$, Mari Odaira ${ }^{1}$, \\ Masanobu Yoshida ${ }^{1}$, Kazuki Shiina ${ }^{1}$, Mikio Nagata ${ }^{4}$ and Akira Yamashina ${ }^{1}$
}

We examined whether the central aortic systolic blood pressure, a marker of the function of the systemic arterial tree, might be a more powerful predictor of the development of hypertension than the brachial-ankle pulse wave velocity, a marker of the stiffness of the large- to middle-sized arteries, independent of the conventional risk factors for the development of hypertension. In 1268 Japanese men without hypertension ( $43 \pm 8$ years old), the relationships between three variables (the second peak of the radial pressure waveform (SP2), brachial-ankle pulse wave velocity and conventional risk factors measured at the first examination) with the presence of hypertension at the second examination (after 3 years' follow-up) were examined.

Hypertension was detected at the second examination in 154 men. The best cutoff points of the brachial-ankle pulse wave velocity and SP2, for predicting the development of hypertension, were $12.7 \mathrm{~m} / \mathrm{s}$ and $109 \mathrm{~mm} \mathrm{Hg}$, respectively. The results of a logistic regression analysis confirmed that an SP2 of $\geqslant 109 \mathrm{~mm} \mathrm{Hg}$ (odds ratio $=8.493, P<0.001$ ) was a more powerful predictor of the development of hypertension than a brachial-ankle pulse wave velocity of $\geqslant 12.7 \mathrm{~m} / \mathrm{s}$, independent of the conventional risk factors. The net reclassification index of SP2 (at the best cutoff point) to brachial-ankle pulse wave velocity was $0.211(P<0.001)$, indicating that SP2 is a better predictor of the development of hypertension than brachial-ankle pulse wave velocity. In middle-aged Japanese men without hypertension, SP2 may be a more powerful predictor of the development of hypertension than the assessment of stiffness in large to middle-sized arteries independent of the conventional risk factors. Hypertension Research (2013) 36, 19-24; doi:10.1038/hr.2012.123; published online 9 August 2012

Keywords: arterial stiffness; central blood pressure; hypertension; pressure wave reflection; prospective study

\section{INTRODUCTION}

Vascular mechanisms are known to have vital roles in the development of hypertension. ${ }^{1}$ Some prospective studies have demonstrated that the markers related to stiffness in conduit arteries are predictors of the development of hypertension, ${ }^{2-6}$ and we previously demonstrated that the brachial-ankle pulse wave velocity (brachial-ankle PWV), a marker of the stiffness of the large- to middle-sized arteries, might be a reliable predictor of the future development of hypertension. ${ }^{4}$ On the other hand, central aortic systolic pressure (CASP) is a parameter of the central hemodynamics, and not only abnormalities of the conduit arteries but also those of the peripheral arteries affect the CASP. ${ }^{7-10}$ The vascular statuses of both the conduit and the peripheral arteries have been suggested to have pivotal roles in elevation of the blood pressure. ${ }^{1}$ Thus, it was considered possible that the CASP is a more powerful predictor of the development of hypertension than the assessment of abnormalities of conduit arteries. However, no prospective study has been conducted to verify this hypothesis.

The present prospective study was conducted to clarify whether the second peak of the radial pressure wave form (SP2), a marker of CASP, ${ }^{7,8,11,12}$ might be a more powerful predictor of the development of hypertension than the brachial-ankle PWV in middle-aged Japanese men without hypertension, independent of the conventional risk factors for the development of hypertension such as age, obesity, smoking status, alcohol intake, brachial blood pressure, and heart rate.

\section{METHODS}

\section{Subjects}

This prospective study was conducted in a cohort of Japanese employees of the company headquarters of a single large construction company. ${ }^{4,13}$ The subjects underwent an annual health checkup in 2007, The examinations, including blood pressure measurements (two times) with the subjects in the seated position, blood and urine examinations, and measurements of the brachialankle PWV and radial augmentation index (radial AI), were conducted in the morning after the subjects had fasted overnight. Among the study subjects, those who were working at the company headquarters were invited to undergo follow-up measurements of the brachial-ankle PWV and radial AI, and also blood and urine examinations in 2010. Informed consent was obtained from all the study participants before their participation in this study. The study complies with the Declaration of Helsinki and was conducted with the

\footnotetext{
${ }^{1}$ Second Department of Internal Medicine, Tokyo Medical University, Tokyo, Japan; ${ }^{2}$ St Vincent's Clinic, Sydney, NSW, Australia; ${ }^{3}$ Department of Health Economics and Epidemiology Research, University of Tokyo School of Public Health, Tokyo, Japan and ${ }^{4}$ Health Care Center, Kajima Corporation, Tokyo, Japan 
approval of the Ethical Guidelines Committee of the Tokyo Medical University. The follow-up protocol is described in detail elsewhere. ${ }^{4,13}$

Subjects fulfilling the following criteria either at the baseline (2007) or at the final examination (2010) were excluded from the study: (1) ankle/brachial systolic blood pressure index of less than 0.95 ; (2) presence of atrial fibrillation; (3) serum c-reactive protein (CRP) level exceeding $10.0 \mathrm{mg} / \mathrm{l}$ (as the conventional clinical cutoff point for inflammation); (4) value of the s.d. of the radial $\mathrm{AI}$, as calculated from ten radial pressure waveform records, of $\geqslant 6 \% .^{12}$ In addition, subjects receiving medications for heart disease, stroke, and/or risk factors for cardiovascular diseases (that is, hypertension, hypercholesterolemia and/or diabetes mellitus) at the baseline examination were also excluded.

In the year 2007, 2412 subjects working at the headquarters of a construction company were enrolled in this follow-up study. Of these, 2185 subjects were successfully followed up until 2010, while the remaining 227 subjects were recorded as lost to follow-up because of their failure to visit the center for completion of the testing or other reasons (moving from the company headquarters to branch offices, change of job, retirement, and so on). Of the 2185 subjects, 220 subjects were initially excluded because of the presence of atrial fibrillation $(n=6)$, ankle/brachial systolic blood pressure index of less than $0.95(n=62)$, values of the s.d. of the radial AI of $\geqslant 6 \%$ $(n=116)$, or serum CRP levels exceeding $10.0 \mathrm{mg} / \mathrm{l} \quad(n=36)$. Then, the remaining 1965 subjects were enrolled for the follow-up examinations. At the start of the follow-up period, 212 subjects with a history of treatment for heart disease, stroke, and/or risk factors for cardiovascular disease were excluded. Among these, 182 were labeled as having hypertension and 1268 subjects as having normal blood pressure (all the 293 women were excluded, because their number was relatively small as compared with the number of men included in the study).

\section{Measurements}

Brachial blood pressure measurement and definition of the blood pressure category. Brachial blood pressure was determined as the mean of two measurements obtained in an office setting by the conventional cuff method using a mercury sphygmomanometer. These two measurements were performed on the same occasion with the subjects in the seated position after at least $5 \mathrm{~min}$ of rest. Then, according to the mean of the duplicate brachial blood pressure measurements, the subjects were labeled as having normal blood pressure (less than $140 / 90 \mathrm{~mm} \mathrm{Hg}$ ) or hypertension $(\geqslant 140 / \geqslant 90 \mathrm{~mm} \mathrm{Hg}$ ). Subjects receiving antihypertensive medications were categorized as having hypertension.

Alcohol use. Habitual alcohol use was assessed using a self-administered questionnaire. The alcohol use level was evaluated using two parameters: the average drinking frequency (days/week) and the average amount consumed every week $(\mathrm{ml})$. The average daily alcohol use level (g/day, ethanol equivalent) was then calculated for each subject. Then, the subjects were categorized according to their daily alcohol use, estimated from their responses to the questionnaire, as follows: no history of alcohol consumption, no-alcohol group; consumes $1-29 \mathrm{~g}$ of alcohol/day, light-to-moderate alcohol use group; consumes $>30 \mathrm{~g}$ of alcohol/day, heavy alcohol use group. ${ }^{4}$

Pulse wave velocity. Brachial-ankle PWV was measured using a volumeplethysmographic apparatus (Form/ABI, Colin, Komaki, Japan), in accordance with previously described methodology. ${ }^{4,13}$ In brief, occlusion cuffs, which were connected to both the plethysmographic and oscillometric sensors, were tied around both the upper arms and ankles of the subjects lying in the supine position. The brachial and post-tibial arterial pressures were measured using the oscillometric sensor. The measurements were conducted after the subjects had rested for at least $5 \mathrm{~min}$ in the supine position, in a temperature-controlled room $\left(24-26^{\circ} \mathrm{C}\right)$ designated exclusively for this purpose.

Augmentation index. Measurements of the blood pressure and radial AI were conducted after the subjects had rested for at least $5 \mathrm{~min}$ in the sitting position. The blood pressure was measured in the right upper arm using the oscillometric method (HEM-907; Omron Healthcare, Kyoto, Japan). Immediately after this measurement, the left radial arterial waveform was recorded using an arterial applanation tonometry probe equipped with an array of 40 micropiezo-resistive transducers (HEM-9010AI; Omron Healthcare). The HEM-9010AI device is programmed to automatically determine the pressure of the radial artery to yield the optimal radial arterial waveform. ${ }^{11,12}$ Then, the first and second peaks of the peripheral systolic pressure (SP1 and SP2, a marker of the CASP) and peripheral diastolic pressure (DP) were automatically determined using the fourth derivatives for each radial arterial waveform, and averaged. The radial AI, a marker of the central AI, was calculated as follows: $(\mathrm{SP} 2$ - brachial DP $) /\left(\mathrm{SP} 1\right.$ - brachial DP) $\times 100(\%) .{ }^{11,12}$

\section{Laboratory measurements}

Fasting serum concentrations of triglycerides, total cholesterol, high-density lipoprotein cholesterol and creatinine, and plasma glucose (FPG) concentrations were measured using enzymatic methods (Falco Biosystems, Tokyo, Japan). The blood and urine samples were collected at the time of the annual health checkups. The serum CRP level was determined by the latex-aggregation method (Eiken, Tokyo, Japan), which is a high-sensitivity assay method with a detection threshold of $<0.1 \mathrm{mg} / \mathrm{l}$.

\section{Statistical Analyses}

Data were expressed as means \pm s.d. The differences in the measured values between the first and second examinations were assessed by the paired $t$-test. McNemar's non-parametric test was applied for assessment of the differences in the categorical variables between the first and second examinations.

A logistic regression analysis was performed to assess the relationship between the variables measured at the first examination and the presence of hypertension at the second examination. Among the variables conventionally known as risk factors for the development of hypertension, those with a significant crude odds ratio for the presence of hypertension at the second examination were entered as covariates for the adjustments in the multivariable logistic regression analysis with brachial-ankle PWV, radial AI, SP1 and SP2 at the first examination as main explanatory variables. Then, the adjusted odds ratios of the variables for the presence of hypertension at the second examination were determined.

The values obtained at the second examination minus those obtained at the first examination were determined as the delta changes of the brachial systolic and diastolic blood pressures during the study period. The relationships among the variables were assessed by univariate linear regression analysis and also multivariate linear regression analysis with adjustments for covariates (the covariates were the same as those entered in the logistic regression analysis).

A receiver-operating characteristic curve analysis was conducted to compare performance of the brachial-ankle PWV and SP2 recorded at the first examination to predict hypertension at the second examination and also to identify the best cutoff point of these two variables. In receiver-operating characteristic curve analysis, the best cutoff point of the variables was determined by the Youden index (Sensitivity + Specificity -1). ${ }^{14}$ Then, the variables were dichotomized at the cutoff point, and were tested by logistic regression analysis with adjustments for covariates. Finally, the net reclassification index of the best cutoff point of the SP2 to that of the brachial-ankle PWV at the first examination for predicting the development of hypertension was calculated. ${ }^{15}$

All of the analyses were conducted using the IBM/SPSS software for Windows, version $19.0 \mathrm{~J}$ (IBM/SPSS, Chicago, IL, USA); $P$-values $<0.05$ were considered to denote statistical significance.

\section{RESULTS}

During the 3-year follow-up period, 154 men developed hypertension by the time of the second examination (Among these, 35 had been initiated on antihypertensive medication by the time of the second examination). Table 1 depicts the clinical characteristics of the study subjects at the first and second examinations. Significant increases of the body mass index (BMI), alcohol intake, blood pressure variables, brachial-ankle PWV, and parameters of the central hemodynamics from the first to the second examination were observed.

Table 2 depicts the baseline clinical characteristics of the study subjects with and without development of hypertension by the end of 
Table 1 Clinical characteristics of the study subjects (number of study subjects $=1268$ )

\begin{tabular}{|c|c|c|c|}
\hline Parameter & First examination & Second examination & P-value \\
\hline Age & $43 \pm 8$ & & \\
\hline $\mathrm{BMI}$ & $23.7 \pm 2.8$ & $23.9 \pm 2.8$ & $<0.001$ \\
\hline Smoking (\%) & $387(31)$ & $339(27)$ & $<0.001$ \\
\hline Alc (non/mimo/heav) (\%) & $220 / 718 / 330$ & $162 / 627 / 479$ & $<0.001$ \\
\hline $\mathrm{BSP}(\mathrm{mm} \mathrm{Hg})$ & $120 \pm 10$ & $121 \pm 12$ & $<0.001$ \\
\hline $\mathrm{BDP}(\mathrm{mm} \mathrm{Hg})$ & $72 \pm 9$ & $75 \pm 9$ & $<0.001$ \\
\hline HR (beats/min) & $68 \pm 9$ & $67 \pm 10$ & $<0.001$ \\
\hline baPWV (m/s) & $12.5 \pm 1.3$ & $12.9 \pm 1.6$ & $<0.001$ \\
\hline $\operatorname{rAl}(\%)$ & $69.0 \pm 13.2$ & $72.9 \pm 12.5$ & $<0.001$ \\
\hline $\mathrm{SP} 1(\mathrm{~mm} \mathrm{Hg})$ & $121 \pm 12$ & $122 \pm 13$ & 0.011 \\
\hline $\mathrm{SP} 2(\mathrm{~mm} \mathrm{Hg})$ & $107 \pm 14$ & $109 \pm 14$ & $<0.001$ \\
\hline $\mathrm{TC}(\mathrm{mmol} / \mathrm{l})$ & $5.34 \pm 0.86$ & $5.39 \pm 0.83$ & 0.008 \\
\hline $\mathrm{TG}(\mathrm{mmol} / \mathrm{l})$ & $1.39 \pm 1.00$ & $1.37 \pm 1.03$ & 0.369 \\
\hline $\mathrm{HDL}(\mathrm{mmol} / \mathrm{l})$ & $1.62 \pm 0.40$ & $1.67 \pm 0.42$ & $<0.001$ \\
\hline $\mathrm{FPG}(\mathrm{mmol} / \mathrm{l})$ & $5.03 \pm 0.53$ & $4.98 \pm 0.59$ & $<0.001$ \\
\hline Crnn $(\mu \mathrm{mol} / \mathrm{l})$ & $76.49 \pm 9.80$ & $76.81 \pm 9.52$ & 0.095 \\
\hline CRP (mg/l) & $0.93 \pm 1.16$ & $0.90 \pm 1.02$ & 0.319 \\
\hline \multicolumn{4}{|c|}{$\begin{array}{l}\text { Abbreviations: BMI, body mass index; Alc (non/mimo/heav), non, non-drinker; mimo, mild to } \\
\text { moderate drinker; heav, heavy drinker; BSP, brachial systolic blood pressure; BDP, brachial } \\
\text { diastolic blood pressure; HR, heart rate determined from the electrocardiogram at the time of } \\
\text { measurement of the brachial-ankle pulse wave velocity; baPWV, brachial-ankle pulse wave } \\
\text { velocity; rAI, radial augmentation index; SP1, first peak of the radial pressure waveform; } \\
\text { SP2, second peak of the radial pressure waveform; TC, serum total cholesterol; HDL, serum } \\
\text { high-density lipoprotein cholesterol; TG, serum triglycerides; FPG, fasting plasma glucose; } \\
\text { Crnn, serum creatinine level; CRP, serum level of C-reactive protein }\end{array}$} \\
\hline
\end{tabular}

Table 2 Baseline clinical characteristics of the study subjects with and without hypertension

\begin{tabular}{|c|c|c|c|}
\hline Parameter & $\begin{array}{l}\text { Without hypertension } \\
\qquad(\mathrm{n}=1114)\end{array}$ & $\begin{array}{l}\text { With hypertension } \\
\qquad(\mathrm{n}=154)\end{array}$ & P-value \\
\hline Age & $42 \pm 8$ & $47 \pm 8$ & $<0.001$ \\
\hline BMI & $23.6 \pm 2.7$ & $24.7 \pm 3.0$ & $<0.001$ \\
\hline Smoking (\%) & 328 (29) & $59(38)$ & 0.034 \\
\hline $\begin{array}{l}\text { Alc }(\text { non/mimo/ } \\
\text { heav) }(\%)\end{array}$ & $203 / 644 / 267$ & $17 / 74 / 63$ & $<0.001$ \\
\hline $\mathrm{BSP}(\mathrm{mm} \mathrm{Hg})$ & $119 \pm 10$ & $128 \pm 9$ & $<0.001$ \\
\hline $\mathrm{BDP}(\mathrm{mm} \mathrm{Hg})$ & $71 \pm 8$ & $79 \pm 8$ & $<0.001$ \\
\hline HR (beats/min) & $65 \pm 8$ & $68 \pm 9$ & $<0.001$ \\
\hline baPWV (m/s) & $12.4 \pm 1.2$ & $13.5 \pm 1.5$ & $<0.001$ \\
\hline rAl (\%) & $68.0 \pm 13.1$ & $75.5 \pm 12.0$ & $<0.001$ \\
\hline $\mathrm{SP} 1(\mathrm{~mm} \mathrm{Hg})$ & $120 \pm 11$ & $134 \pm 12$ & $<0.001$ \\
\hline SP2 (mm Hg) & $105 \pm 12$ & $122 \pm 14$ & $<0.001$ \\
\hline $\mathrm{TC}(\mathrm{mmol} / \mathrm{l})$ & $5.33 \pm 0.87$ & $5.39 \pm 0.78$ & 0.494 \\
\hline $\mathrm{TG}(\mathrm{mmol} / \mathrm{l})$ & $1.36 \pm 0.99$ & $1.59 \pm 1.06$ & 0.014 \\
\hline $\mathrm{HDL}(\mathrm{mmol} / \mathrm{l})$ & $1.62 \pm 0.40$ & $1.59 \pm 0.41$ & 0.339 \\
\hline $\mathrm{FPG}(\mathrm{mmol} / \mathrm{l})$ & $5.01 \pm 0.53$ & $5.19 \pm 0.53$ & $<0.001$ \\
\hline Crnn $(\mu \mathrm{mol} / \mathrm{l})$ & $76.48 \pm 9.77$ & $76.60 \pm 10.03$ & 0.888 \\
\hline CRP (mg/l) & $0.93 \pm 1.19$ & $0.94 \pm 1.02$ & 0.918 \\
\hline
\end{tabular}

Abbreviations are described in the footnote for Table 1.

the study period. Significant differences in age, BMI, brachial systolic and diastolic blood pressures, heart rate, serum triglycerides levels, FPG levels, smoking status, and alcohol consumption status were observed between the two groups.

Table 3 shows the results of the logistic regression analysis performed to determine the crude odds ratio and $95 \%$ confidence intervals $(95 \% \mathrm{CI})$ for the variables measured at the first examination
Table 3 Logistic regression analysis to determine the crude odds ratio and $95 \%$ confidence intervals for the variables measured at the first examination to predict the development to hypertension

\begin{tabular}{lccr}
\hline Variable & Crude odds ratio & $95 \%$ Cl & P-value \\
\hline Age 1st & 1.072 & $1.050-1.094$ & $<0.001$ \\
BMI 1st & 1.139 & $1.076-1.207$ & $<0.001$ \\
Smoking 1st & 1.488 & $1.049-2.111$ & $<0.001$ \\
Alc 1st & 1.795 & $1.371-2.350$ & $<0.001$ \\
BSP 1st & 1.112 & $1.088-1.137$ & $<0.001$ \\
BDP 1st & 1.125 & $1.097-1.153$ & $<0.001$ \\
HR 1st & 1.050 & $1.031-1.070$ & $<0.001$ \\
baPWV 1st & 1.006 & $1.004-1.007$ & $<0.001$ \\
rAI 1st & 1.047 & $1.032-1.062$ & $<0.001$ \\
SP1 1st & 1.114 & $1.095-1.134$ & $<0.001$ \\
SP2 1st & 1.105 & $1.088-1.122$ & $<0.001$ \\
TC 1st & 1.065 & $0.877-1.294$ & 0.526 \\
TG 1st & 1.198 & $1.043-1.377$ & 0.011 \\
HDL 1st & 0.809 & $0.526-1.243$ & 0.333 \\
FPG 1st & 1.647 & $1.258-2.157$ & $<0.001$ \\
Crnn 1st & 1.001 & $0.984-1.019$ & 0.886 \\
logCRP 1st & 1.378 & $0.763-2.489$ & 0.288 \\
\hline Abbration & & &
\end{tabular}

Abbreviations: 1st, at the first examination; 95\% Cl, 95\% confidential interval; logCRP, logtransformed plasma C-reactive protein levels; other abbreviations are explained in the footnote for Table 1.

to predict the presence of hypertension at the second examination. Age, BMI, brachial systolic and diastolic blood pressures, heart rate, serum triglycerides levels, FPG levels, smoking status, and alcohol consumption status showed significant crude odds ratios for the presence of hypertension at the second examination. Then, these variables were entered as covariates for the adjustments in the multiple logistic regression analysis. Each of the brachial-ankle PWV, radial AI, SP1 and SP2 at the first examination showed a significant crude odds ratio for the presence of hypertension at the second examination (Table 3 ).

After the adjustments, each of the brachial-ankle PWV, radial AI, SP1 and SP2 at the first examination was identified to show a significant adjusted odds ratio for the presence of hypertension at the second examination (Table 4A). Then, to examine the independency of these variables to predict the presence of hypertension at the second examination, all the variables were entered. Only the SP2 on the first examination remained significant as an independent predictor of the development of hypertension (Table 4B).

In the univariate linear regression analysis, the brachial-ankle PWV $(r=0.072, P=0.010)$ and SP2 $(r=0.105, P<0.001)$ measured at the first examination were found to be significantly correlated with the delta change of the brachial systolic blood pressure during the study period. The SP2 $(r=0.075, P<0.001)$, but not the brachial-ankle PWV ( $r=0.041, P=0.141)$, measured at the first examination was found to be significantly correlated with the delta change of the brachial diastolic blood pressure during the study period. Then, a multivariate linear regression analysis, with the brachial-ankle PWV and SP2 measured at the first examination entered into the analysis model simultaneously with the variables used as covariates for the adjustments in the logistic regression analysis, demonstrated that both the brachial-ankle PWV \{non-standardized coefficient $=0.009(95 \%$ $\mathrm{CI}=0.006-0.012)$; standardized coefficient $=0.101, P=0.001\}$ and SP2 \{non-standardized coefficient $=0.257(95 \% \mathrm{CI}=0.230-0.284)$; standardized coefficient $=0.299, P<0.001\}$ measured at the first examination showed a significant independent relationship with the 
Table 4 (A and B) Assessment of the independency of the variables for predicting the development of hypertension during the observation period by logistic regression analysis with adjustments

\begin{tabular}{|c|c|c|c|}
\hline Variable & Adjusted odds ratio & $95 \% \mathrm{Cl}$ & P-value \\
\hline \multicolumn{4}{|c|}{$\begin{array}{l}\text { A: Each of the variables (baPWV 1st, rAI 1st, SP1 1st and SP2 1st) was separatel, } \\
\text { entered into the model of logistic regression analysis with adjustments }\end{array}$} \\
\hline baPWV 1st & 1.003 & $1.001-1.004$ & $<0.001$ \\
\hline rAl 1st & 1.053 & $1.033-1.073$ & $<0.001$ \\
\hline SP1 1st & 1.087 & 1.065-1.109 & $<0.001$ \\
\hline SP2 1st & 1.085 & $1.065-1.105$ & $<0.001$ \\
\hline \multicolumn{4}{|c|}{$\begin{array}{l}\text { B: Each of the variables (baPWV } 1 s t, r A I 1 s t, S P 11 s t \text { and SP2 1st) was } \\
\text { simultaneously entered into the model of logistic regression analysis with } \\
\text { adjustments }\end{array}$} \\
\hline baPWV 1st & 0.999 & 0.998-1.001 & 0.491 \\
\hline rAl 1st & 0.974 & $0.915-1.037$ & 0.408 \\
\hline SP1 1st & 0.975 & 0.882-1.078 & 0.625 \\
\hline SP2 1st & 1.126 & $1.006-1.261$ & 0.039 \\
\hline
\end{tabular}

Abbreviation: Abbreviations are explained in the footnote for Table 1; the covariates for the adjustments were the conventional risk factors with significant crude odds ratios to predict the development of hypertension (age, BMI, systolic and diastolic blood pressures, heart rate, serum TG levels, FPG levels, smoking status, and alcohol use status).

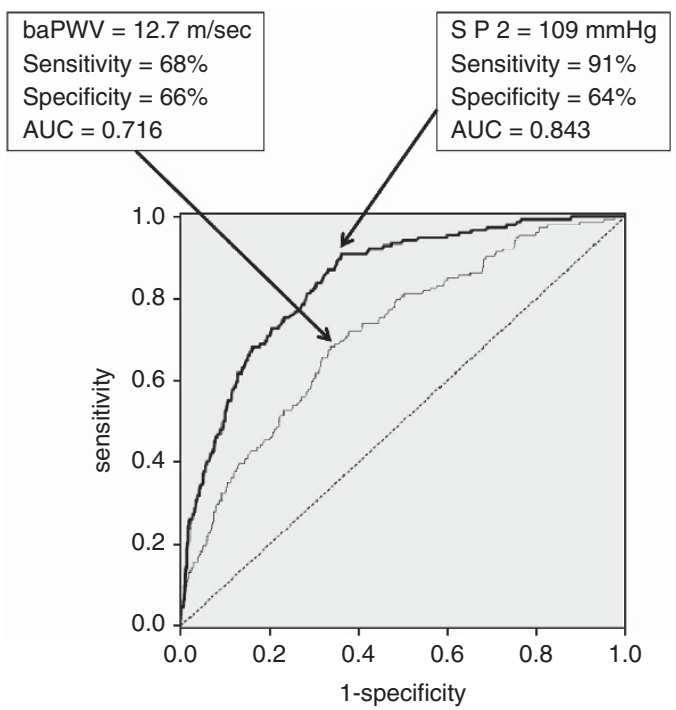

Figure 1 Receiver-operator characteristic curves to illustrate the relationship between the variables measured at the first examination and the progression to hypertension during the study period. AUC, area under the curve; SP2, second peak of the radial pressure waveform at the first examination.

delta change of the brachial systolic blood pressure during the study period $\left(R^{2}=0.344\right)$. Further, the SP2 measured at the first examination (non-standardized coefficient $=0.233(95 \% \mathrm{CI}=0.211-0.255)$; standardized coefficient $=0.336, P<0.001)$, but not the brachialankle PWV measured at the first examination \{non-standardized coefficient $=0.000 \quad(95 \% \quad \mathrm{CI}=-0.001 \quad$ to 0.002$) ; \quad$ standardized coefficient $=0.006, P=0.827\}$, showed a significant independent relationship with the delta change of the brachial diastolic blood pressure during the study period $\left(R^{2}=0.343\right)$.

Estimated areas under the curve to predict the presence of hypertension at the second examination were as follows: brachialankle PWV at the first examination $=0.716(95 \% \mathrm{CI}=0.674-0.759)$ and SP2 at the first examination $=0.843(95 \% \mathrm{CI}=0.812-0.874)$. As
Table 5 The calculation of the net reclassification index of the best cutoff point of the SP2 to that of the brachial-ankle PWV measured at the first examination for predicting the presence of hypertension

Reclassification included SP2

Non-case Case

Subjects without developed hypertension by the time of the second examination $(\mathrm{n}=1114)$

The assessment with conventional risk factors including baPWV

$\begin{array}{lll}\text { Non-case } & 547 & 181 \\ \text { Case } & 163 & 223\end{array}$

Subjects with developed hypertension by the time of the second examination $(\mathrm{n}=154)$

The assessment with conventional risk factors including baPWV

$\begin{array}{lll}\text { Non-case } & 6 & 43 \\ \text { Case } & 8 & 97\end{array}$

The upward and downward movements in the subjects without developed hypertension were 181 and 163, respectively, and the corresponding movements in the subjects with developed hypertension were 43 and 8 , respectively. Thus, the net reclassification index was 0.211 , $P<0.001$.

shown in Figure 1, the best cutoff points of the brachial-ankle PWV and SP2 determined at the first examination for predicting the presence of hypertension at the second examination were $12.7 \mathrm{~m} / \mathrm{s}$ (sensitivity $=68 \%$ and specificity $=66 \%$; Youden index $=0.34$ ) and $109 \mathrm{~mm} \mathrm{Hg} \quad$ (sensitivity $=91 \%$ and specificity $=64 \%$; Youden index $=0.55$ ). When brachial-ankle PWV $>12.7 \mathrm{~m} / \mathrm{s}$ at the first examination and SP2 $\geqslant 109 \mathrm{mmHg}$ at the first examination were entered separately into the logistic regression analysis model with adjustments for conventional risk factors, both were significantly predictive of the presence of hypertension at the second examination. However, when both were entered simultaneously into this logistic regression model, SP2 $\geqslant 109 \mathrm{~mm} \mathrm{Hg}$ at the first examination (adjusted odds ratio $=8.493,95 \% \mathrm{CI}=4.629-15.582, P<0.001$ ), but not the brachial-ankle PWV at the first examination (adjusted odds ratio $=1.293,95 \% \mathrm{CI}=0.838-1.994, P=0.246$ ), was significantly predictive of the presence of hypertension at the second examination.

The net reclassification index of the best cutoff point of the SP2 to that of conventional risk factors, including the brachial-ankle PWV measured at the first examination, for predicting the presence of hypertension was $0.211(P<0.001)$ (Table 5).

\section{DISCUSSION}

This is the first prospective study to clarify whether SP2, a marker of CASP, might be a reliable predictor of the development of hypertension.

\section{Previous findings to predict the development of hypertension} Previous studies have demonstrated that age, brachial blood pressure levels, heart rate, BMI, FPG levels, serum CRP levels, alcohol intake status and smoking status are factors that influence the risk of development of hypertension. ${ }^{4,16}$ In this study, after adjustments for these factors, the brachial-ankle PWV and SP2 were identified to show significant odds ratios for the new onset of hypertension. When the significance of these two variables was compared, the SP2 rather than the brachial-ankle PWV was identified to show a significant odds 
ratio for the development of hypertension. Furthermore, the net reclassification index has been proposed as a useful index to evaluate the strengths of new markers to improve the risk stratification of subjects for cardiovascular disease; ${ }^{14}$ in this study, the net reclassification index of the SP2 to the brachial-ankle PWV was 0.211 . Thus, this prospective study suggested that the SP2 served as a more reliable marker for predicting the development of hypertension than the brachial-ankle PWV, independent of the conventional risk factors for the development of hypertension.

\section{Previous findings of CASP as a marker of cardiovascular risk}

Several prospective studies have demonstrated that the CASP is an independent risk factor for the future development of cardiovascular events. ${ }^{17,18}$ On the other hand, physiological markers have also been focused on as markers of the early stage pathophysiological abnormalities related to the risk of cardiovascular disease, such as subclinical organ damage. ${ }^{8,19-21}$ McEniery et al. demonstrated that more than $70 \%$ of subjects with high normal blood pressure had similar CASP levels as the subjects with stage I hypertension; therefore, they proposed assessment of the CASP to improve the identification and management of patients with an elevated risk of cardiovascular diseases. ${ }^{22}$ The results of the present study suggesting that SP2 is an independent risk factor for the development of hypertension is in line with the concept that the CASP can serve as an independent marker of the early-stage pathophysiological abnormalities related to increased cardiovascular risk.

\section{CASP and the development of hypertension}

Previous studies have demonstrated that carotid arterial elasticity and aortic elasticity as assessed by ultrasound examination, brachial-ankle PWV, a marker of stiffness of the large to middle-sized arteries, and carotid-femoral PWV, a marker of stiffness of the large artery, are predictors of the development of hypertension. ${ }^{2-6}$ Therefore, functional abnormalities in the arteries may be associated with the development of hypertension. The plausible underlying mechanisms are as follows: (1) increase in aortic impedance elevates the systolic blood pressure; (2) the attenuated cushioning effect of the aorta increases the propagation of the pressure energy generated by cardiac contraction to the peripheral organs. Then, microvascular damage caused by this increased propagation pressure elevates the blood pressure via increasing the peripheral vascular resistance and/or causing renal function decline. ${ }^{2-5,20,21}$

On the other hand, CASP is a marker of the central hemodynamics, and pressure wave reflection is one of the major determinants of the CASP; the latter is not only affected by the status of the conduit arteries, but also by that of the peripheral arteries. ${ }^{7-10}$ Recently, Perelta et al. suggested that reduced small arterial elasticity is the earliest predictor of the development of hypertension. ${ }^{6}$ In addition to the influence of the conduit-arterial status, abnormal peripheral vascular status directly elevates the blood pressure via several mechanisms, such as by inducing endothelial dysfunction and increasing the peripheral vascular resistance. ${ }^{1,23}$

Finally, an increase in the blood pressure itself within the normal range, such as prehypertension, is a risk factor for the development of hypertension. ${ }^{24}$ In the present study, among the variables related to arterial stiffness, central hemodynamics and blood pressure, SP2 was the most powerful predictor of the development of hypertension. Therefore, CASP might be a comprehensive marker representing several pathophysiological abnormalities related to the development of hypertension.

\section{Limitations}

The present study had some limitations, as follows: (1) in the present study, the blood pressure categories were defined by the duplicate brachial blood pressure measurements obtained on the day of the annual health check-up. Therefore, there is the possibility of diminished accuracy of the blood pressure categorization, but we did not have any scope to resolve this issue in this prospective study; (2) we could not examine the effects of habitual exercise, which is known to affect the risk of development of hypertension; (3) the significance of CASP as a predictor of the development of hypertension should be confirmed in the elderly, in female subjects and in subjects of other ethnicities.

\section{CONCLUSION}

In middle-aged Japanese men without hypertension, SP2 may be a more powerful predictor of the development of hypertension than the assessment of stiffness in large to middle-sized arteries, independent of the conventional risk factors for the development of hypertension. Thus, functional abnormalities in the systemic arterial tree may have some roles in the increased risk of hypertension, even in the early stage of cardiovascular disease.

\section{CONFLICT OF INTEREST}

The authors declare no Conflict of interest

\section{ACKNOWLEDGEMENTS}

This study was partially supported by the fund of J-CHEARS (Japan Cohort of Hypertensive Elderly: Arterial Stiffness) Study. This study was supported in part by a fund from OMRON Health Care (Kyoto, Japan).

1 Kaplan NM. Primary hypertension: pathogenesis. In: Kaplan NM and Victor RG (eds) Kaplan Primary Hypertension. Wolter Kluter/Lippincott Williams \& Wilkins, Philadelphia, PA, 2010, pp 42-107.

2 Liao D, Arnett DK, Tyroler HA, Riley WA, Chambless LE, Szklo M, Heiss G. Arterial stiffness and the development of hypertension. The ARIC study. Hypertension 1999; 34: 201-206.

3 Dernellis J, Panaretou M. Aortic stiffness is an independent predictor of progression to hypertension in nonhypertensive subjects. Hypertension 2005; 45: 426-431.

4 Tomiyama H, Matsumoto C, Yamada J, Yoshida M, Odaira M, Shiina K, Nagata M, Yamashina A. Predictors of progression from prehypertension to hypertension in Japanese men. Am J Hypertens 2009; 22: 630-636.

5 Najjar SS, Scuteri A, Shetty V, Wright JG, Muller DC, Fleg JL, Spurgeon HP, Ferrucci L, Lakatta EG. Pulse wave velocity is an independent predictor of the longitudinal increase in systolic blood pressure and of incident hypertension in the Baltimore Longitudinal Study of Aging. J Am Coll Cardiol 2008; 51: 1377-1383.

6 Peralta CA, Adeney KL, Shlipak MG, Jacobs Jr D, Duprez D, Bluemke D, Polak J, Psaty B, Kestenbaum BR. Structural and functional vascular alterations and incident hypertension in normotensive adults: the multi-ethnic study of atherosclerosis. Am J Epidemiol 2010; 171: 63-71.

7 O'Rourke MF, Hashimoto J. Mechanical factors in arterial aging: a clinical perspective. J Am Coll Cardiol 2007; 50: 1-13.

8 Tomiyama H, Yamashina A. Non-invasive vascular function tests: their pathophysiological background and clinical application. Circ J 2010; 74: 24-33.

9 Nichols WW. Clinical measurement of arterial stiffness obtained from noninvasive pressure waveforms. Am J Hypertens 2005; 18(1 Pt 2): 3S-10S.

10 Vlachopoulos C, Aznaouridis K, O'Rourke MF, Safar ME, Baou K, Stefanadis C. Prediction of cardiovascular events and all-cause mortality with central haemodynamics: a systematic review and meta-analysis. Eur Heart J 2010; 31: 1865-1871.

11 Melenovsky V, Borlaug BA, Fetics B, Kessler K, Shively L, Kass DA. Estimation of central pressure augmentation using automated radial artery tonometry. J Hypertens 2007; 25: 1403-1409.

12 Tomiyama H, Yamazaki M, Sagawa Y, Teraoka K, Shirota T, Miyawaki Y, Yamashina A. Synergistic effect of smoking and blood pressure on augmentation index in men, but not in women. Hypertens Res 2009; 32: 122-126.

13 Tomiyama H, Hashimoto H, Tanaka H, Matsumoto C, Odaira M, Yamada J, Yoshida M, Shiina K, Nagata M, Yamashina A. Continuous smoking and progression of arterial stiffening: a prospective study. J Am Coll Cardiol 2010; 55: 1979-1987.

14 Fluss R, Faraggi D, Reiser B. Estimation of the Youden Index and its associated cutoff point. Biom J. 2005; 47: 458-472. 
15 Pencina MJ, D’Agostino Sr RB, D’Agostino Jr RB, Vasan RS. Evaluating the added predictive ability of a new marker: from area under the ROC curve to reclassification and beyond. Stat Med 2008; 27: 157-172.

16 Niskanen L, Laaksonen DE, Nyyssönen K, Punnonen K, Valkonen VP, Fuentes R, Tuomainen TP, Salonen R, Salonen JT. Inflammation, abdominal obesity, and smoking as predictors of hypertension. Hypertension 2004; 44: 859-865.

17 Pini R, Cavallini MC, Palmieri V, Marchionni N, Di Bari M, Devereux RB, Masotti G, Roman MJ. Central but not brachial blood pressure predicts cardiovascular events in an unselected geriatric population: the ICARe Dicomano Study. J Am Coll Cardiol 2008; 51: 2432-2439.

18 Wang KL, Cheng HM, Sung SH, Chuang SY, Li CH, Spurgeon HA, Ting CT, Najjar SS, Lakatta $\mathrm{EG}$, Yin FC, Chou $\mathrm{P}$, Chen $\mathrm{CH}$. Wave reflection and arterial stiffness in the prediction of 15-year all-cause and cardiovascular mortalities: a community-based study. Hypertension 2010; 55: 799-805.

19 Halcox JP, Donald AE, Ellins E, Witte DR, Shipley MJ, Brunner EJ, Marmot MG, Deanfield JE. Endothelial function predicts progression of carotid intima-media thickness. Circulation 2009; 119: 1005-1012.
20 Tomiyama H, Tanaka H, Hashimoto H, Matsumoto C, Odaira M, Yamada J, Yoshida M, Shiina K, Nagata M, Yamashina A. Arterial stiffness and declines in individuals with normal renal function/early chronic kidney disease. Atherosclerosis 2010; 212: 345-350.

21 Munakata M, Miura Y, Yoshinaga K. J-TOPP study group. Higher brachial-ankle pulse wave velocity as an independent risk factor for future microalbuminuria in patients with essential hypertension: the J-TOPP study. J Hypertens 2009; 27: 1466-1471.

22 McEniery CM, Yasmin, McDonnell B, Munnery M, Wallace SM, Rowe CV, Cockcroft JR, Wilkinson IB. Anglo-Cardiff Collaborative Trial Investigators. Central pressure: variability and impact of cardiovascular risk factors: the Anglo-Cardiff Collaborative Trial II. Hypertension 2008; 51: 1476-1482.

23 Soga J, Nakamura S, Nishioka K, Umemura T, Jitsuiki D, Hidaka T, Teragawa H, Takemoto H, Goto C, Yoshizumi M, Chayama K, Higashi Y. Relationship between augmentation index and flow-mediated vasodilation in the brachial artery. Hypertens Res 2008; 31: 1293-1298.

24 Redwine KM, Acosta AA, Poffenbarger T, Portman RJ, Samuels J. Development of hypertension in adolescents with pre-hypertension. J Pediatr 2012; 160: 98-103. 